To appear in Astrophysical Journal March 2009-v693 issue

\title{
The Spitzer c2d Survey of Nearby Dense Cores. V. Discovery of a VeLLO in the "Starless" Dense Core L328
}

\author{
Chang Won Lee ${ }^{1,2}$, Tyler L. Bourke ${ }^{2}$, Philip C. Myers², Mike Dunham ${ }^{3}$, Neal Evans ${ }^{3}$, \\ Youngung Lee ${ }^{1}$, Tracy Huard ${ }^{4}$, Jingwen $\mathrm{Wu}^{2}$, Robert Gutermuth ${ }^{2}$, Mi-Ryang Kim ${ }^{1}$, Hyun \\ Woo Kang ${ }^{1}$ \\ ${ }^{1}$ Korea Astronomy \&5 Space Science Institute, 61-1 Hwaam-dong, Yusung-gu, Daejeon \\ 305-348, Korea.E-mail: cwl@kasi.re.kr \\ ${ }^{2}$ Harvard-Smithsonian Center for Astrophysics, 60 Garden Street, Cambridge, MA 02138, \\ USA \\ ${ }^{3}$ Department of Astronomy, The University of Texas at Austin, 1 University Station, \\ C1400, Austin, Texas 78712-0259, USA \\ ${ }^{4}$ Department of Astronomy, The University of Maryland, College Park, MD 20742-2421, \\ $U S A$
}

\begin{abstract}
This paper reports the discovery of a Very Low Luminosity Object (VeLLO) in the "starless" dense core L328, using the Spitzer Space Telescope and ground based observations from near-infrared to millimeter wavelengths. The Spitzer 8 $\mu \mathrm{m}$ image indicates that L328 consists of three subcores of which the smallest one may harbor a source, L328-IRS while two other subcores remain starless. L328IRS is a Class 0 protostar according to its bolometric temperature $(44 \mathrm{~K})$ and the high fraction $(\sim 72 \%)$ of its luminosity emitted at sub-millimeter wavelengths. Its inferred "internal luminosity" $\left(0.04-0.06 \mathrm{~L}_{\odot}\right)$ using a radiative transfer model under the most plausible assumption of its distance as $200 \mathrm{pc}$ is much fainter than for a typical protostar, and even fainter than other VeLLOs studied previously. Note, however, that its inferred luminosity may be uncertain by a factor of $2-3$ if we consider two extreme values of the distance of L328-IRS (125 or $310 \mathrm{pc}$ ). Low angular resolution observations of $\mathrm{CO}$ do not show any clear evidence of a molecular outflow activity. But broad line widths toward L328, and Spitzer and near-infrared images showing nebulosity possibly tracing an outflow cavity, strongly suggest the existence of outflow activity. Provided that an envelope of
\end{abstract}


at most $\sim 0.1 \mathrm{M}_{\odot}$ is the only mass accretion reservoir for L328-IRS, and the star formation efficiency is close to the canonical value $\sim 30 \%$, L328-IRS has not yet accreted more than $0.05 \mathrm{M}_{\odot}$. At the assumed distance of $200 \mathrm{pc}$, L328-IRS is destined to be a brown dwarf.

Subject headings: ISM: individual (L328, L328-IRS)-stars: formation: low-mass, brown-dwarfs

\section{Introduction}

Knowledge of how low mass stars form has been significantly improved thanks to the recent availability of large telescopes and sensitive detectors, especially at millimeter and infrared wavelengths. Molecular cloud condensations are understood to gradually increase in density via several processes such as ambipolar diffusion or turbulent dissipation with some contribution of magnetic fields. When the condensations are dense enough to be detected with high density tracers such as the CS and $\mathrm{NH}_{3}$ transitional lines, but have not yet formed a protostar, they are called "starless" cores (e.g., Benson \& Myers 1989, Lee, Myers, \& Tafalla 1999). Some starless cores are believed to be gravitationally bound, and are called pre-stellar cores under the assumption that protostars are likely to form in the future (e.g., Ward-Thompson et al. 2007, Di Francesco et al. 2007). Once a core become gravitationally unstable and collapses, it forms a protostar (the main accretion stage) with a disk and infalling envelope. The protostar then evolves to the stage where the envelope has mostly dispersed and a young stellar object (YSO) with an optically thick disk is revealed, and eventually the disk becomes optically thin to finally reveal a normal main-sequence (MS) star (e.g., André et al. 2000, Robitaille et al. 2006). Even though we have a good consensus on the global scenario of evolution from the cores to MS stars, we still have very little knowledge about which conditions decide how stars form and evolve in the dense cores. Probably the initial stage of star formation may play the most important role in its further evolution. This is why we study "starless" dense cores.

Dense molecular cores have typically been classified as "starless" if they contain no Infrared Astronomical Satellite (IRAS) point source whose flux increases in the longer wavelengths (Beichmann et al. 1986; Benson \& Myers 1989; Lee \& Myers 1999). Therefore the definition of "starless" has been limited to the sensitivity of the IRAS of $\sim 0.1(\mathrm{~d} / 140)^{2} \mathrm{~L}_{\odot}$ where $\mathrm{d}$ is the distance to the source (Myers et al. 1987). This implies that studying protostars embedded in dense cores has been limited only to sources brighter than $0.1 \mathrm{~L}_{\odot}$ at the distance of nearby star-forming regions such as Taurus, resulting in fewer studies of young stars fainter than $0.1 \mathrm{~L}_{\odot}$ forming in the cores. 
The sensitivity of the Spitzer Space Telescope (hereafter Spitzer) at infrared wavelengths allows, for the first time, sensitive explorations to detect deeply embedded faint objects in dense cores that were not detected by IRAS or the Infrared Space Observatory (ISO) (Young et al. 2004; Dunham et al. 2006; Bourke et al. 2006). Such objects may become protostars or brown dwarfs depending on how they obtain matter as time goes on. The objects fainter than $0.1 \mathrm{~L}_{\odot}$ are called Very Low Luminosity Objects (VeLLOs) (Young et al. 2004, Di Francesco et al. 2007; Dunham et al. 2008). The key feature of these sources is that their luminosity is an order of magnitude fainter than the accretion luminosity $\left(\sim 1.6 \mathrm{~L}_{\odot}\right)$ that the lowest mass protostar of $\sim 0.08 \mathrm{M}_{\odot}$ can produce, under the standard star formation theory (e.g., Shu et al. 1987), with a typical accretion rate $\left(\sim 2 \times 10^{-6} \mathrm{M}_{\odot} \mathrm{yr}^{-1}\right)$ and protostellar radius of $\sim 3 \mathrm{R}_{\odot}$ (Dunham et al. 2006). These may simply be either very faint protostars that will grow to become normal young stars, or proto-brown dwarfs that eventually become brown dwarfs. So far there is no clear conclusion about what these objects are and will be in the future because of the limited number of VeLLOs studied. There are only three VeLLOs studied in detail: L1014-IRS (Young et al. 2004), L1521F-IRS (Bourke et al. 2006), and IRAM 04191+1522 (Dunham et al. 2006). Their properties are similar in terms of their faintness $\left(0.06-0.09 \mathrm{~L}_{\odot}\right)$, but found to be different in their outflow activity and relations with physical properties of their parent cloud cores (Bourke et al. 2006, Dunham et al. 2008). Detailed studies on more VeLLOs are needed to better understand these objects and their role in star formation.

This paper presents a study of another VeLLO, L328-IRS in the dark cloud Lynds 328, using observations performed with a part of Spitzer c2d Legacy project (Evans et al. 2003, 2007) together with several complementary ground-based observations. L328-IRS has been listed as one of 15 VeLLO candidates that were found in a search of c2d cores and clouds by Dunham et al. (2008).

L328 is a dense molecular cloud located in projection between two nearby large clouds, the Ophiuchus Molecular Cloud (hereafter the Ophiuchus) and the Aquila Rift. In L328, neither an IRAS point source nor any PMS stars had been found, and thus it was classified as "starless" (Lee \& Myers 1999). Therefore this object has been studied as one of the starless dense cores by many researchers (Visser et al. 2001, 2002; Bacmann et al. 2002, 2003; Crapsi et al. 2005b; Löhr et al. 2007). The one thing to note is that the line width of $\mathrm{N}_{2} \mathrm{H}^{+}$1-0 is broad $\left(\Delta \mathrm{V}=0.438 \mathrm{~km} \mathrm{~s}^{-1}\right)$ compared with other starless cores, indicating the existence of the significant contribution of turbulent gaseous motion (Crapsi et al. 2005b). Crapsi et al. (2005b) have classified L328 as "not chemically and dynamically evolved" because it does not show any evolved feature such as infall asymmetry, significant CO depletion, or deuteration. 
The distance to L328 is an important issue for this study. We will explain how we adopt the distance of L328 in $\S 2$. In $\S 3$ we describe Spitzer observations as well as other groundbased observations. The images of L328, photometric properties of L328-IRS, association of L328-IRS with L328, and outflow activity of L328-IRS are described in $\S 4$. The Spectral Energy Distribution (SED) of L328-IRS is modeled with a radiative transfer code and the fate of L328-IRS is discussed in $§ 5$. The summary can be found in the last section.

\section{Distance of L328}

L328 is one of the c2d cores whose distance is not clearly known. This is mainly because the association of L328 with either of the two nearby large clouds whose distances are reasonably well known, the Ophiuchus and the Aquila Rift, is not clear. The spatial location on the sky of L328 at $l, b=13^{\circ} 03,-0^{\circ} 83$ is between the Ophiuchus located at approximately $-12^{\circ}<1<12^{\circ}$ and $10^{\circ}<\mathrm{b}<25^{\circ}$ and the Aquila Rift located at approximately $20^{\circ}<1<40^{\circ}$ and $-6^{\circ}<\mathrm{b}<14^{\circ}$ (Dame et al. 1987, 2001, Straižys et al. 2003). Interestingly, the systemic velocity $\left(\mathrm{V}_{\mathrm{LSR}}=6.5 \mathrm{~km} \mathrm{~s}^{-1}\right.$ from this study) of L328 is also somewhat in between the Ophiuchus ( $\left.\mathrm{V}_{\mathrm{LSR}}=-2 \sim 7 \mathrm{~km} \mathrm{~s}^{-1}\right)$ and the Aquila Rift $\left(\mathrm{V}_{\mathrm{LSR}}=2 \sim 12 \mathrm{~km} \mathrm{~s}^{-1}\right.$ ) (Dame et al. 2001). Considering all aspects, it is likely that L328 is located at a distance between Ophiuchus and Aquila.

There are recent measurements of the distance of the Ophiuchus cloud using parallax observations of radio emission of young stars (Loinard et al. 2008; $120 \pm 4.5 \mathrm{pc}$ ) and by combining extinction maps and parallaxes from Hipparcos and Tycho (Mamajek 2008; $135 \pm$ 8pc, Lombardi et al. 2008;119 \pm 6 pc). We adopt the distance of the Ophiuchus cloud as $125 \mathrm{pc}$ in our paper which is the same distance as that of de Geus et al. (1989) and also consistent with other measurements and the distance used in previous c2d papers (Young et al. 2006; Enoch et al. 2007; Padgett et al. 2008). The distance of the central region of the Aquila Rift has been estimated as 270 pc using interstellar extinction of stars over the cloud (Straižys et al. 2003), By taking an average of the distances of two clouds, we assume the distance of L328 core as 200 pc in the paper. The Aquila Rift has been suggested to have different distances for front (225 pc) and far (310 pc) edges (Straižys et al. 2003), We will discuss a possibility for L328 to be located at the far edge of the Aquila Rift and its effect on our results. 


\section{Observations}

The infrared observations toward L328 from 3.6 to $70 \mu \mathrm{m}$ were conducted as a part of the c2d Legacy program (Evans et al. 2003) using Spitzer equipped with the Infrared Array Camera (IRAC; Fazio et. 2004) and the Multi-band Imaging Photometer (MIPS; Rieke et al. 2004). The IRAC observations were made in four bands centered on 3.6, 4.5, 5.8, and $8.0 \mu \mathrm{m}$. In order to remove contamination from asteroids, observations were performed in two epochs with about a day gap. The first epoch observation with IRAC was made on 2004 September 3 (AOR key 5147136) and the second on 2004 September 4 (AOR key 5147392). Each observation has four dithers of 12 seconds each, offset by $\sim 10^{\prime \prime}$ to cover about a $5^{\prime} \times 5^{\prime}$ field with a total integration time of $48 \mathrm{~s}$ for each image.

The MIPS images were obtained in two bands of 24 and $70 \mu \mathrm{m}$ in two epochs to detect and remove the asteroids. The first epoch observation was made on 2004 April 11 (AOR key 9420544) and the second one on 2004 April 12 (AOR key 9436160). Observations were made in MIPS photometry mode with a sky offset of 300". The total integration time for 24 and $70 \mu \mathrm{m}$ observations for each epoch was $48 \mathrm{~s}$ and $126 \mathrm{~s}$, respectively.

After identifying an infrared source in L328, we also conducted follow-up deeper observations (called as Cores2Deeper) toward L328 with Spitzer (PID: 20386, PI: P. Myers), using IRAC, and MIPS at $24 \mu \mathrm{m}$. The observation with IRAC was made on 2005 September 20 (AOR key 14608384) and the observation with MIPS at $24 \mu \mathrm{m}$ was made on 2005 September 26 (AOR key 14616064), respectively. The IRAC images were obtained with two epoch observations, 8 dithers of $30 \mathrm{~s}$ per epoch. Therefore the on source time for IRAC image in each bands was $480 \mathrm{~s}$ which is 10 times longer than $\mathrm{c} 2 \mathrm{~d}$ data, making the images 3.2 times deeper than c2d data. For MIPS 24, we made one epoch observation with an on source time of $333 \mathrm{~s}$. Thus the MIPS $24 \mu \mathrm{m}$ image had 6.9 times longer on source time or 2.6 times better sensitivity than the $\mathrm{c} 2 \mathrm{~d}$ data. In data analysis for this study we used the Cores2Deeper data from 3.6 to $24 \mu \mathrm{m}$ and c2d data at $70 \mu \mathrm{m}$.

The IRAC and MIPS images were processed by the Spitzer Science Center through their standard pipeline version S13. The c2d team improved the images by correcting bad pixels and other artifacts and made photometry of the extracted sources with a modified version of DOPHOT (Schechter et al. 1993) using a digitized point source profile. More details on the improvement of the images are given in the documentation for $\mathrm{c} 2 \mathrm{~d}$ data delivery by Evans et al. (2007).

We also tried to observe or retrieve data for L328 at the other wavelengths bands to broaden our knowledge of L328. Molecular line observations in the 3-mm band were made with the Seoul Radio Astronomy Observatory (SRAO) 6 m radio telescope in 2005 April. 
L328 was observed over the area of $16^{\prime} \times 16^{\prime}$ with $1^{\prime}$ or $2^{\prime}$ grid spacings in the CO (1-0) line, over $4^{\prime} \times 4^{\prime}$ area with $1^{\prime}$ grid spacings in $\mathrm{C}^{18} \mathrm{O}(1-0), 2^{\prime} \times 2^{\prime}$ area with $1^{\prime}$ grid spacings in $\mathrm{CS}(2$ 1 ), and $4^{\prime} \times 4^{\prime}$ area with $1^{\prime}$ grid spacings in $\mathrm{N}_{2} \mathrm{H}^{+}(1-0)$. In the $\mathrm{CO}$ (1-0) line observations the FWHM beam size of the SRAO was $\sim 100^{\prime \prime}$ (Koo et al. 2002), the velocity resolution was $\sim 0.13 \mathrm{~km} \mathrm{~s}^{-1}$, and the typical rms sensitivity in $\mathrm{T}_{\mathrm{A}}^{*}$ unit was $\sim 0.2 \mathrm{~K}$.

The NIR (J, H, \& Ks) data from the PANIC IR camera of Baade 6.5m telescope at Las Campanas (Huard et al. 2008 in prep.), $350 \mu \mathrm{m}$ continuum data from SHARC-II on the CSO (Wu et al. 2007), $850 \mu \mathrm{m}$ continuum data from JCMT SCUBA (Di Francesco et al. 2008), and $1.2 \mathrm{~mm}$ data from the bolometer array of the IRAM 30-m telescope (Bacmann et al. 2000) were also obtained for this paper.

\section{Results}

\subsection{Images of L328}

Fig. 1 shows a Digital Sky Survey (DSS) red image of L328 over an area of $\sim 16^{\prime} \times 16^{\prime}$. The figure shows a dark opaque core (L328) of $\sim 2^{\prime} \times 2^{\prime}$ size and several curved long tails which extend to the SW $\sim 15$ arc-minute long (L327). Fig. 2 shows Spitzer images of the region indicated by a white box in Fig. 1, for reference, with its DSS-red image with $350 \mu \mathrm{m}$ emission contours overlaid. At optical wavelengths L328 is very opaque, and thus no background stars are seen towards L328. However, our Spitzer observations are able to penetrate the deep dark regions of L328 to see both embedded or background stars. L328 becomes significantly transparent at 3.6 and $4.5 \mu \mathrm{m}$ so that a number of background stars are seen. Starlight gets fainter in longer wavelength bands such as 5.8 and $8.0 \mu \mathrm{m}$. However, background emission from $6.2 \& 8.6 \mu \mathrm{m}$ features of Polycyclic Aromatic Hydrocarbons (PAHs) becomes dominantly bright at these wavelengths because L328 is located almost toward the Galactic plane. This allows L328 to be seen in absorption against the bright background at 5.8 and $8.0 \mu \mathrm{m}$. L328 is also dark at $24 \mu \mathrm{m}$. There is no feature of the PAH emission at this band and the bright background is most likely due to hot dust emission over the Galactic disk plane.

Note that L328 at 5.8, 8.0 and $24 \mu \mathrm{m}$ is not seen as a single simple dark core, but as two main dark regions and one small dark region in the South. These sub-structures in L328 have been also found in $350 \mu \mathrm{m}$ dust emission by $\mathrm{Wu}$ et al. (2007) who designated these as L328-smm1, smm2, and smm3 from West to East. Their $350 \mu \mathrm{m}$ image indicates that L328-smm1 is the biggest among three, L328-smm3 is the next, and L328-smm2 is the smallest in size. 
The $70 \mu \mathrm{m}$ image of L328 is not shown due to its low angular resolution. The L328 core is seen in absorption at $70 \mu \mathrm{m}$ which is rare (Stutz al. al 2008) but only as a single core.

\subsection{L328-IRS}

\subsubsection{Photometric properties of L328-IRS}

A surprise in the Spitzer observations is a discovery of an infrared source (L328-IRS) at the SW edge of L328. The position of L328-IRS is marked with an arrow in Fig. 2. L328-IRS is rather bright at 3.6, 4.5, and $5.8 \mu \mathrm{m}$, gets dimmer in $8.0 \mu \mathrm{m}$, and then much brighter at 24 and $70 \mu \mathrm{m}$ than any other sources in the L328 region. This property, increasing flux to longer wavelengths, suggests that L328-IRS may be a protostar.

The interesting thing to note is that it does not look like a point source, but is slightly extended at the short wavelengths $(3.6,4.5$, and $5.8 \mu \mathrm{m})$. The JHKs image with the highest angular resolution, shown in Fig. 3, indicates that this is partly because the source itself has extended emission and also partly because the Spitzer images of L328-IRS are possibly contaminated with two nearby sources about $1^{\prime \prime}-2^{\prime \prime}$ apart located to the South of the source. We find that in the Ks image L328-IRS consists of a nucleus and an extended nebulosity, and the position of the nucleus is consistent with the position of L328-IRS in all Spitzer images. Thus the position of L328-IRS is given as the position of the nucleus seen in Ks image $\left[(\alpha, \delta)_{J 2000}=\left(18^{\mathrm{h}} 16^{\mathrm{m}} 59.50,-18^{\circ} 02^{\prime} 30^{\prime \prime} .5\right)\right]$ which is determined as the flux weighted centroid of the nucleus part of the Ks image.

Because L328-IRS is somewhat extended, the automated photometry by c2d team utilizing a point source profile was not reliable for this particular source. This required us to perform aperture photometry for this object at each wavelength using the "PHOT" task in IRAF to include all emitting parts of the source. For the photometry in IRAC and MIPS bands, we made an aperture correction in its values by using correction factors provided by Spitzer Science Center. For the photometry at sub-millimeter wavelengths, although it was not so clear which aperture size is the most appropriate, we tried to include all the emission around smm2 sub-core, but not to include emission from the two other sub-cores, smm1 and smm3. We chose a compromise aperture size of $20^{\prime \prime}$ for this reason and measured emission fluxes from the dusty envelope surrounding L328-IRS at 350, 850, and $1200 \mu \mathrm{m}$. The measured fluxes and apertures for each wavelength are listed in Table 1.

This enables us to construct the SED for L328-IRS and to measure its infrared slope,

$\alpha\left(\alpha=\frac{\log \lambda F_{\lambda}}{\log \lambda}\right.$; Lada \& Wilking 1984, Lada 1987). The slope is calculated to be 0.61 $( \pm 0.63)$ from a linear least squares fit for the range of $3.6-24 \mu \mathrm{m}$, indicating that L328- 
IRS may be a Class I protostar ( $\alpha$ is not defined for Class 0 protostars). The bolometric temperature, which is defined as the temperature of a blackbody having the same fluxweighted mean frequency as the observed continuum spectrum (Myers \& Ladd 1993), is 44 $\mathrm{K}$, and the fraction of luminosity at sub-millimeter wavelengths $(\geq 350 \mu \mathrm{m})$ with respect to the bolometric luminosity is found to be about 72 percent. These additional quantities are consistent with L328-IRS being a Class 0 type protostar.

\subsubsection{Association of L328-IRS with L328}

Fig. 4 shows a three color composite image around L328-IRS in $5.8 \mu \mathrm{m}$ (blue), $8.0 \mu \mathrm{m}$ (green), and $24 \mu \mathrm{m}$ (red). This map shows sub-structures of L328 and illustrates how they are related to L328-IRS. Three sub-cores appear to be embedded in one dusty envelope and two of them, smm1 (NW) and smm3 (NE), seem to be connected in the northern parts while between these two cores in the south there is a cavity structure seemingly starting from L328-IRS, which may be related to outflow activity from L328-IRS or simply a region of lower column density. Further south there is one smaller core (smm2), that is connected to smm1 by a curved dusty lane. L328-IRS is most closely aligned with smm2, with its position offset by $\sim 3^{\prime \prime}$ from the peak position of smm2.

Note that L328-IRS is only prominently red source in the region. The colors of all point sources in L328 field observed with the Spitzer are shown in Fig. 5, where L328-IRS is distinctively red in [5.8]-[24.0] color $([3.6]-[4.5]=1.21$ and [5.8]-[24.0]=5.94) while all other objects are $<1$ in [5.8]-[24.0] color. It is interesting to note that the colors of other known VeLLOs are similarly red in [5.8]-[24.0] color (4.66 for L1014-IRS, 5.94 for IRAM04191, and 7.67 for L1521F-IRS). We also found that the magnitude at $24 \mu \mathrm{m}\left(\mathrm{m}_{24}=5.05\right)$ and [8.0]-[24] color of 6.35 for L328-IRS are far out of the likely range of these values for the extragalactic SWIRE sources (see Fig. 3 of Harvey et al. 2007), indicating that L328-IRS is unlikely to be a background galaxy. Taken all together, L328-IRS is associated with high column density material and is likely to be deeply embedded within smm2.

Fig. 6 shows molecular line profiles toward L328-IRS. Note that the position where the profiles were taken is accidentally $\sim 27.5^{\prime \prime}$ offset from L328-IRS. However, since the SRAO beam size is large enough (100" FWHM at the frequency of CO 1-0), these line data should be useful to characterize L328-IRS and its environment. The CO profile indicates that there are several clouds with at least five different LSR velocities of 6.5, 12.0, 17.8, 43.4 and 64 $\mathrm{km} \mathrm{s}^{-1}$ along the line of sight of L328-IRS. Only two of those components, 6.5 and 43.4 $\mathrm{km} \mathrm{s}^{-1}$, are detected in the denser tracers such as $\mathrm{C}^{18} \mathrm{O}$ (1-0) and CS (2-1) lines, and only the $6.5 \mathrm{~km} \mathrm{~s}^{-1}$ component is detected in $\mathrm{N}_{2} \mathrm{H}^{+}(1-0)$ line. Note that $\mathrm{N}_{2} \mathrm{H}^{+}(1-0)$ emission has 
been previously detected from single pointing observations by Lee, Myers, \& Tafalla (1999) and Crapsi et al. (2005b). $\mathrm{C}^{18} \mathrm{O}$ integrated line maps of the velocity components at 6.5 and $43.4 \mathrm{~km} \mathrm{~s}^{-1}$ show that the $6.5 \mathrm{~km} \mathrm{~s}^{-1}$ component is a local peak corresponding to the dark opaque part of L328, while there are no clear condensations of the $43.4 \mathrm{~km} \mathrm{~s}^{-1}$ component and therefore no hint of an association of L328-IRS with the $43 \mathrm{~km} \mathrm{~s}^{-1}$ component (Fig. 7). Thus L328-IRS is likely to be associated with the gas at $6.5 \mathrm{~km} \mathrm{~s}^{-1}$ following the tendency for a star to form in a dense molecular region (e.g., Beichmann et al. 1986).

\subsubsection{Outflow activity of L328-IRS}

If L328-IRS is truly a protostar embedded in L328, then we may expect some outflow activity. CO profiles can test for a possible existence of the molecular outflow. However, the CO profile of the $6.5 \mathrm{~km} \mathrm{~s}^{-1}$ velocity component does not show any broad wing component. Moreover, integrated intensity maps of blue-shifted and red-shifted velocities with respect to the systemic velocity do not show any significant difference that would indicate the existence of a molecular outflow. Our CO data do not seem to show any clear evidence of outflows toward L328-IRS.

However, this does not necessarily mean that there is no outflow activity in L328-IRS because there is a possibility that the large beam $\left(100^{\prime \prime}\right)$ of the SRAO might dilute any signature of outflow if it exists at very small scales, just as the case of L1014-IRS where the small scale ( $\sim 500 \mathrm{AU})$ outflow has been found through sensitive interferometry observations with the SMA (Bourke et al. 2005) while it has not been clearly detected with lower angular resolution (Crapsi et al. 2005a).

Near IR H \& Ks observations with high sensitivity and fine angular resolution, shown in Fig. 3, give some hints for the outflow activity around L328-IRS (Huard et al. 2008 in prep.). First of all, we see a relatively empty region (designated as A in Fig. 3) of about 30" in length crossing the central region of L328 from SW to NE which is also seen in the Spitzer images in Fig. 2 and 4. This looks like an elongated cavity shape possibly due to material being evacuated by an outflow activity from L328-IRS. The opposite part of the cavity SW of L328-IRS is, however, not clearly seen probably because there is not enough gaseous material to highlight its relatively tenuous presence compared to surrounding regions.

Second, L328-IRS is the only source having a nebulosity in L328 region. Moreover, L328IRS at Ks band has a structure of a point-like nucleus plus an extended feature of nebulosity to the SW while the overall image of L328-IRS at $\mathrm{H}$ band has only diffuse extended emission which is nearly coincident with the extended part of Ks band image (the right panel of 
Fig. 3). This feature may be related to nebulosity due to scattered light through a cavity associated with outflow activity within a dusty envelope (Huard et al. 2006).

Third, a line bisecting L328-IRS and the $30^{\prime \prime}$ cavity structure (A in Fig. 3) to its NE also passes through the nebulosity (B in Fig. 3) of the protostar itself seen in the near IR, indicating that two different outflow structures are possibly related.

Fourth, line widths of $\mathrm{N}_{2} \mathrm{H}^{+}$toward the central region of $\mathrm{L} 328$ where the cavity structure exists are broader $\left(0.438 \pm 0.005 \mathrm{~km} \mathrm{~s}^{-1}\right.$ from Crapsi et al. 2005b) than for most starless cores $\left(0.24 \pm 0.06 \mathrm{~km} \mathrm{~s}^{-1}\right.$ : an average of FWHMs for the $\mathrm{N}_{2} \mathrm{H}^{+}$profiles of 28 starless cores except for L328 listed in Crapsi et al. 2005b) where thermal motions dominate. Our CO map rules out the possibility that some cloud components with slightly different velocities could cause the broadening of the line profile. Thus the broad line width may be due to some turbulence effects produced by the outflow activity. One thing to note is that the $\mathrm{N}_{2} \mathrm{H}^{+}$line profile by Crapsi et al. (2005b) has not been obtained from the exact position of L328-IRS, but from a position $\left[(\alpha, \delta)_{J 2000}=\left(18^{\mathrm{h}} 17^{\mathrm{m}} 00^{\mathrm{s}} 40,-18^{\circ} 01^{\prime} 52^{\prime \prime}\right.\right.$. $\left.)\right]$ about $40^{\prime \prime}$ offset to NE from L328-IRS. However, the $\mathrm{N}_{2} \mathrm{H}^{+}$observation with the IRAM 30m beam at that position is found to include a significant part of the $30^{\prime \prime}$ cavity structure and thus may characterize the possible outflow effect due to L328-IRS. Therefore, although the $\mathrm{N}_{2} \mathrm{H}^{+}$profile may not directly reflect the physical status toward L328-IRS, its broad line width may be an indirect signature of the possible outflow activity from L328-IRS.

\section{Discussion}

\subsection{Radiative transfer modeling of the SED of L328-IRS}

The observed luminosity (i.e., bolometric luminosity) of L328-IRS was calculated to be about $0.19 \mathrm{~L}_{\odot}$ by using fluxes integrated through the observed wavelength range and its assumed distance of $200 \mathrm{pc}$. This luminosity $\left(\mathrm{L}_{\mathrm{bol}}\right)$ is the sum of an internal luminosity $\left(\mathrm{L}_{\mathrm{int}}\right)$ due to the central source and a luminosity $\left(\mathrm{L}_{\text {isrf }}\right)$ supplied by the interstellar radiation field (ISRF) from the outside. The emission from the envelope is partly caused by heating by the internal luminosity source and partly by the ISRF. Therefore it is necessary to separate the emission of the central source from other contributions to estimate its internal luminosity $\mathrm{L}_{\mathrm{int}}$. This can be done through a detailed study of the radiative process of emission from the central source through the dusty envelope. To do this we used a one dimensional radiative transfer package DUSTY (Ivezic. 1999) to calculate the SED of L328-IRS with assumed physical properties of the central source and its dusty envelope. Similar studies of other VeLLOs have recently been undertaken (Young et al. 2004; Dunham et al. 2006; Bourke 
et al. 2006); all the details of the input parameters to this modeling are described in those studies and Young \& Evans (2005).

Our model consists of a stellar black body, a cooler disk, dusty envelope, and the Interstellar Radiation Field (ISRF). Starting with an input spectrum from the central star and disk, the model calculates the emission reprocessed by the model envelope with an educated guess of the contribution of the ISRF. By adjusting the free parameters in the model, the fitting process is iterated to get the $\chi^{2}$ best fit of the modeled SED to the observed SED, and thus the best fit internal luminosity $\mathrm{L}_{\text {int }}$.

The internal luminosity of the central source in the model is the luminosity from the stellar component plus the luminosity from the disk. For the stellar component, we assume the temperature and luminosity of a blackbody that provide a reasonable fit to the flux densities between $3.6-8.0 \mu \mathrm{m}$. The flux densities at these emergent from the central source, however, are absorbed by the dusty envelope surrounding L328-IRS and reprocessed to longer wavelengths. Especially the flux density at $8 \mu \mathrm{m}$ is highly affected by absorption which may be caused mainly by $9.7 \mu \mathrm{m}$ silicate features in the dusty envelope. These need to be taken into account. The flux densities at 24 and $70 \mu \mathrm{m}$ are not fitted at all by the stellar component, and a dusty disk is included to fit those wavelengths. We employed a model of a disk with surface density and temperature profiles described as $\Sigma(\mathrm{r})=\Sigma_{0}\left(\mathrm{r} / \mathrm{r}_{0}\right)^{-\mathrm{p}}$ and $\mathrm{T}_{\mathrm{r}}=\mathrm{T}_{0}\left(\mathrm{r} / \mathrm{r}_{0}\right)^{-\mathrm{q}}$, respectively (Butner et al. 1994). We adopt the "spherical disk" approach of Butner et al. (1994) in the sense that in the code the emission from the model disk is averaged over all possible viewing angles and then added to the emission from the stellar blackbody to produce an input spectrum for the internal source. In this modeling we tried to fix all free parameters whose variation has small effect on the results. We assume a flared disk with a power index $\mathrm{p}=1.5$ in the surface density distribution, but with a slightly less steep index $(q=0.4)$ in the temperature distribution than in Butner et al. (1994) which helps to better fit the $24 \mu \mathrm{m}$ flux density than a model with $\mathrm{q}=0.5$. The inner radius of the disk was set to be the radius at which dust reaches its sublimation temperature (assumed to be $1500 \mathrm{~K}$ ). It varied from 0.02 to $0.04 \mathrm{AU}$ to be consistent with the internal luminosity of the central source. On the other hand the outer radii were simply fixed to be 50 AU. Usually the model was insensitive with the disk size. We run the models with outer disk radii of 10 AU and $100 \mathrm{AU}$ and found similar results for the inferred internal luminosity to the model with a disk size of $50 \mathrm{AU}$. The code also includes the contribution from intrinsic disk emission which can arise from accretion of the material on the disk.

The input emission emerging at mainly short wavelengths is then reprocessed in the dusty envelope to produce emission at longer wavelengths. We adopted an approximate isothermal equilibrium sphere for the envelope density distribution (Tafalla et al 2002), 
$n(r)=n_{0} /\left[1+\left(r / r_{0}\right)^{\alpha}\right]$, where $n$ is the density, $r$ is the distance from the center, and $n_{0}, r_{0}$, and $\alpha$ are scaling parameters of the density distribution (note that this is not the same $\alpha$ from $\S$ 4.2.1). Alternatively, a power-law density distribution for the envelope $\left[n(r) \propto r^{-\alpha}\right]$ was also tested and it was found that a shallow power index such as $\alpha=1.1$ resulted in a very similar fit to the flux densities at sub-millimeters to that of the approximate isothermal sphere .

The envelope is assumed to be heated by the emergent emission from the central source inside and the ISRF outside. The inner radius of the envelope is set to be $50 \mathrm{AU}$ and its outer radius is 10,000 AU which roughly matches the size of the envelope seen in the DSS image. The outer radius of the envelope is large enough to include the other sub-cores in L328. However, this radius was set to better match the sub-millimeter and its long-ward flux densities of the envelope with the model SED. Our simple model of the envelope considers the flux contribution from the smm2 core only, but not that from other sub-cores.

The dust opacities are those of "OH5 dust" (grains with thin ice mantles) by Ossenkopf \& Henning (1994) which is known to be appropriate for cold dense cores (Evans et al. 2001; Shirley et al. 2005). We adopted the Black-Draine ISRF (Black 1994, Draine 1978; see Evans et al. 2001) and assumed it to be attenuated by dust grains having optical constants of Draine \& Lee (1984) with $A_{V}=3.0$. This extinction was chosen to simulate the situation that L328 is somewhat embedded in diffuse dust between the Ophiuchus and the Aquila Rift clouds.

We tried to fix most of free parameters regarding the envelope so that the estimated mass of the envelope is consistent with the mass $\left(\mathrm{M}_{\text {env }}\right)$ of the envelope determined from the $350 \mu \mathrm{m}$ continuum. The mass was calculated from $\mathrm{M}_{\mathrm{env}}=\frac{\mathrm{S}_{\nu} \mathrm{D}^{2}}{\mathrm{~B}_{\nu}(\mathrm{T}) \kappa_{\nu}}$ where $S_{\nu}$ is the flux density at $350 \mu \mathrm{m}, \mathrm{B}_{\nu}(\mathrm{T})$ is the Planck function at temperature $(\mathrm{T})$, and $\kappa_{\nu}$ is dust opacity. The estimated mass is about $0.05-0.09 \mathrm{M}_{\odot}$ in the range of temperatures of $15-20 \mathrm{~K}$ with a measured flux density of $3.2 \mathrm{Jy}\left(20^{\prime \prime}\right.$ beam) with $\kappa_{\nu}=0.101 \mathrm{~cm}^{-2} \mathrm{~g}^{-1}$ (OH5 dust opacities in Ossenkopf \& Henning 1994). The mass was also estimated using the 850 and $1200 \mu \mathrm{m}$ data and found to be consistent within a factor of 2 with the mass derived from the $350 \mu \mathrm{m}$ flux. We chose the parameters of $\mathrm{n}_{0}=6 \times 10^{5} \mathrm{~cm}^{-3}, r_{0}=1500 \mathrm{AU}$, and $\alpha=2.0$ as reasonable parameters resulting in an envelope mass within a $2000 \mathrm{AU}$ radius of $0.07 \mathrm{M}_{\odot}$, consistent with the observed mass of the envelope of $20^{\prime \prime}$ aperture.

With this combination of parameters, we tried to obtain a best fit with stellar temperatures $\mathrm{T}_{\text {star }}=1500,1800,2000,2500,3000$, and $3500 \mathrm{~K}$ by adjusting $\mathrm{L}_{\text {int }}$. Among these temperatures a central source of $0.04-0.06 \mathrm{~L}_{\odot}$ is found to produce the best fits to the observed SED. Figure 8 shows a result of the best fit of the observed SED for $T_{\text {star }}=2000 \mathrm{~K}$ and $\mathrm{L}_{\text {int }}=0.05 \mathrm{~L}_{\odot}$ which has a minimum value of $\chi^{2}$. This result implies that most of the 
observed luminosity arises from emission at sub-millimeter wavelength regions heated by the ISRF, as modeled by Evans et al. (2001) for starless cores.

Dunham et al (2008) have estimated the internal luminosity of L328-IRS by using an empirical correlation between fluxes at $70 \mu \mathrm{m}$ and internal luminosities in the VeLLOs that were obtained from the calculation of the SED of embedded low luminosity protostars with the two dimensional radiative transfer code RADMC (Dullemond \& Dominik 2004; Crapsi et al. 2008). Their value of $\mathrm{L}_{\text {int }}$ estimated from the $70 \mu \mathrm{m}$ flux is $0.07 \mathrm{~L}_{\odot}$ which is higher than our $\mathrm{L}_{\mathrm{int}}$. However, their assumed distance of $270 \mathrm{pc}$ is larger than our adopted value. If they had used $200 \mathrm{pc}$, then their luminosity would be $\sim 0.04 \mathrm{~L}_{\odot}$, consistent with our value. Therefore our study suggests that, if the distance of L328 is correct, the luminosity of the central source is well below $0.1 \mathrm{~L}_{\odot}$ and qualifies L328-IRS as a VeLLO.

What if the assumed distance of L328 is different from 200 pc ? We calculated the radiative transfer model for two possible extreme distances $125 \mathrm{pc}$ (the distance of the Ophiuchus) and $310 \mathrm{pc}$ (the distance of the far edge of the Aquila), to examine other possible values of the internal luminosity of L328-IRS due to the uncertainty of the distance, with proper scaling of the mass and the size of its envelope at those distances. The internal luminosities for $\mathrm{T}_{\text {star }}=1500$ to $3500 \mathrm{~K}$ are found to be $0.12-0.18 \mathrm{~L}_{\odot}$ for the distance of 310 pc and $0.02-0.03 \mathrm{~L}_{\odot}$ for the distance of $125 \mathrm{pc}$. This indicates that in the extreme scenario that L328 is possibly located at the far edge of Aquila Rift cloud at 310 pc (Straižys et al. 2003), the internal luminosity of L328-IRS can be slightly higher than the borderline for a VeLLO. Therefore a good knowledge of the distance is a key issue in deciding whether faint sources like L328-IRS are of very low luminosity.

There are several pieces of evidence to suggest that our assumed distance of $200 \mathrm{pc}$ for L328 is more reasonable than $310 \mathrm{pc}$ or even $270 \mathrm{pc}$ (the mean distance to the Aquila Rift). First, the velocity of L328 is intermediate between that of Ophiuchus (125 pc) and the Aquila Rift, suggesting an intermediate distance. Second, L328 is seen in absorption against the background emission at the longer Spitzer wavelengths $(5.8-70 \mu \mathrm{m})$. This background emission is likely due to PAH and warm dust directly associated with the Aquila Rift clouds, and strongly suggests that L328 is in the foreground or on the near side of the Rift].

To summarize, with our best estimate of the distance to L328 of $200 \mathrm{pc}$ and the modeling of the SED of L328-IRS with a radiative transfer code, its inferred internal luminosity is 0.04 - $0.06 \mathrm{~L}_{\odot}$ and qualifies it as a VeLLO. At the distance extremes which seem to be unlikely,

\footnotetext{
${ }^{1}$ Our recent measurement of the distance of L328 using near-IR photometry of 2MASS data around L328 gives a distance of $220 \pm 40$ which is very consistent with our adopted distance (Maheswar \& Lee 2008 in prep.).
} 
$\mathrm{L}_{\text {int }}$ can be lower to $0.02 \mathrm{~L}_{\odot}$ and higher up to $0.18 \mathrm{~L}_{\odot}$ In any case, even if $\mathrm{L}_{\text {int }}=0.18 \mathrm{~L}_{\odot}$ for L328-IRS, its luminosity would still be close to the VeLLO cutoff, which is not strongly based on physical properties, and would still be significantly lower than typical protostars. Accurate determination of the distance of L328-IRS and higher dimensional radiative transfer modeling together with a more complete SED are required to better determine the luminosity of L328-IRS.

\subsection{Evolutionary fate of L328-IRS}

Here we discuss the properties of L328-IRS and compare those with the properties of other VeLLOs. First of all, we note that the internal luminosity of L328-IRS is fainter than that of other VeLLOs studied with a similar level of detail, given that all the distances are fully correct (L1014-IRS, $0.09 \mathrm{~L}_{\odot}$; L1521F-IRS, $0.06 \mathrm{~L}_{\odot}$; IRAM 04191+1522, $0.08 \mathrm{~L}_{\odot}$ ).

We saw several possible hints for the outflow of L328-IRS. Nebulosity of a few arcseconds in length and a probable cavity structure a few tens of arc-seconds long are seen in L328. The projected length of the NE cavity from L328-IRS is about 6,000 AU (30"). If we assume that the nebulosity and tenuous cavity structure represent something related to outflow activity, then the total size of the outflow from L328-IRS would be at least 12,000 AU. This is larger than the outflow from L1014-IRS (a few hundred AU; Bourke et al. 2005), but comparable to the projected size $(\sim 10,000 \mathrm{AU})$ of nebulosity seen in the Spitzer image of L1521F-IRS (Bourke et al. 2005), and much smaller than the pc scale CO outflow in IRAM $04191+1522$.

We did not find any clear evidence that the outflow of L328-IRS may occur in an episodic fashion. We looked for any possibility of an episodic event of the outflow by searching an area of $30^{\prime} \times 30^{\prime}$ around L328 in DSS images and $5^{\prime} \times 5^{\prime}$ in Spitzer images and found no clear evidence of Herbig-Haro objects or shocked region indicating possible episodic events within the outflow activity.

Toward L328 no infall signatures are observed in molecular lines, no significant CO depletion exists (there is $\mathrm{CO}$ depletion, but it is not classified as significant compared to many other cores; Bacmann et al. 2002; Crapsi et al. 2005), and no enhanced deuteration is observed, implying that it is dynamically and chemically young (Crapsi et al. 2005)1. This is somewhat similar to what is found in L1014, but very different from the cores hosting the

\footnotetext{
${ }^{1}$ The molecular line observations referred in this discussion have been made at $(\alpha, \delta)_{J 2000}=\left(18^{\mathrm{h}} 16^{\mathrm{m}} 599^{\mathrm{s}} 50,-18^{\circ} 02^{\prime} 30^{\prime \prime} \cdot 5\right)$ which is about $40^{\prime \prime}$ offset from the position of L328-IRS and closer to smm1 and smm3. Therefore the discussion may rely on the assumption that similarities of the line
} 
VeLLOs, IRAM 04191+1522 and L1521F-IRS, that show many of these properties (André et al. 1999; Belloche et al. 2002; Crapsi et al. 2004).

The mass (at most of order $0.1 \mathrm{M}_{\odot}$ ) of the parent core smm2 associated with L328-IRS as a reservoir providing gaseous material to it is much smaller than other cores with VeLLOs (L1014-IRS, $1.7 \mathrm{M}_{\odot}$, Young et al. 2004; L1521F-IRS, $4.8 \mathrm{M}_{\odot}$, Crapsi et al. 2005; IRAM 04191+1522, $2.5 \mathrm{M}_{\odot}$, Dunham et al. 2006).

Considering these points, L328-IRS is most likely a very low luminosity object, possibly having outflow activity, embedded in an envelope of small mass that is not dynamically or chemically evolved. Is L328-IRS just a small version of a protostar which will eventually grow to a protostar by accreting material from its envelope, or is L328-IRS already on a different pathway to become a brown dwarf? We find that its observed envelope mass is too small as a provider of material for L328-IRS to become a star. If we assume a 30 percent star formation efficiency in L328 (Lada et al. 2007), only a further $\sim 0.03 \mathrm{M}_{\odot}$ of the observed envelope mass will accrete onto L328-IRS.

Therefore, unless other sub-cores in L328 are involving in the accretion process, the star formation efficiency is unusually higher than the canonical value, and it has already accreted more than $\sim 0.05 \mathrm{M}_{\odot}$, L328-IRS seems to have no hope to become a star in the future, and is probably destined to be a brown dwarf. However, if any of these assumptions is not true, alternative scenario in which L328-IRS will grow as a faint protostar and evolve to a normal star is not ruled out either.

\section{Summary}

Using data from Spitzer and complementary data at other wavelengths, we have discovered a very faint infrared source (L328-IRS) toward L328 dark cloud which was previously believed to be starless. L328-IRS is projected onto the smallest submm sub-core in size among three sub-cores identified in the $8 \mu \mathrm{m}$ image, and its infrared spectral slope $(\alpha=0.6)$ and infrared colors strongly suggests that it is embedded within this core. The low bolometric temperature of about $44 \mathrm{~K}$ and the high luminosity fraction (about 72 percent) at sub-millimeter wavelengths $(\geq 350 \mu \mathrm{m})$ with respect to the bolometric Luminosity suggests that L328-IRS is a Class 0 protostar. L328-IRS is by far the reddest object in L328 region.

Our molecular line observations find that the optically obscured part of L328 is associated with molecular gas with a velocity (LSR) of $\sim 6.5 \mathrm{~km} \mathrm{~s}^{-1}$, strongly suggesting an 
association or similar distance to the nearby molecular clouds of Ophiuchus and the Aquila Rift. Based on this information and the absorption of L328 at Spitzer wavelengths against the background emission, we assume a most probable distance of $200 \mathrm{pc}$.

Using the 1-D radiative transfer code "DUSTY" we modeled the observed SED of L328 by assuming that the emission is from a central star of 1500 to $3500 \mathrm{~K}$ with a flared disk that is reprocessed through an isothermal spherical envelope. The key result of this modeling is that the internal luminosity of L328-IRS is in the range of $0.04-0.06 \mathrm{~L}_{\odot}$. This classifies L328IRS as a VeLLO (Di Francesco et al. 2007; Dunham et al. 2008), and of the four confirmed VeLLOs studied to a similar level of detail, L328-IRS is the faintest. However, because two extreme values (125 or $310 \mathrm{pc}$ ) of the distance of L328-IRS may be possible, our calculation with these two distances indicates that its inferred luminosity can be uncertain by a factor of $2-3$. Accurate determination of the distance of L328-IRS would be necessary to confirm whether it is a real VeLLO. Nevertheless its low luminosity even at the farthest plausible distance is still an interesting feature.

The possibility of the existence of a molecular outflow from L328-IRS was explored using $\mathrm{CO}$ observations, but no evidence was found. However, features such as the tenuous cavity structure extending to the NE from L328-IRS at infrared wavelengths, nebulosity extending to the SW from L328-IRS seen in $\mathrm{H}$ and Ks images, and unusually broad line widths toward the central region of L328 in dense gas tracers, lead us to believe that outflow activity is present in L328-IRS. Like the situation in L1014-IRS, the molecular outflow may be too small and weak to be detected by our low angular resolution observations (Bourke et al. 2005).

The parent core around L328-IRS does not appear to be evolved chemically and dynamically, and is too small in mass to enable L328-IRS to become a normal star. Unless the star formation efficiency in the core is abnormally high, other sub-cores within L328 become involved in the growth of L328-IRS, or L328-IRS has already accreted enough mass, L328-IRS is probably destined to become a brown dwarf.

We thank an anonymous referee for useful comments and suggestions. We also thank H. Kirk for providing unsmoothed SCUBA archive data, A. Bacmann for the $1200 \mu \mathrm{m}$ data, and H.J. Kim for the discussion on the DUSTY fitting. CWL \& YL acknowledge the support by the Basic Research Program (KOSEF R01-2003-000-10513-0) of the Korea Science and Engineering Foundation. Support for this work, part of the Spitzer Legacy Science Program, was provided by NASA through contract 1224608 issued by the Jet Propulsion Laboratory, California Institute of Technology, under NASA contract 1407. Partial support for TLB was also provided by NASA through contract 1279198 issued by the Jet Propulsion Laboratory, 
California Institute of Technology, under NASA contract 1407. Partial support was also obtained from NASA Origins grants NNG04GG24G and NNX07AJ72G to NJE.

\section{REFERENCES}

André, P., Motte, F., \& Bacmann, A. 1999, ApJ, 513, L57

André, P., Ward-Thompson, D., \& Barsony, M. 2000, in Protostars and Planets IV, ed. Mannings, V., Boss, A.P., \& Russell, S.S.(Tucson: University of Arizona Press), 59

Bacmann, A., André, P., Pujet, J.-L., Abergel, A., Bontemps, S., \& Ward-Thompson, D. 2000, A\&A, 361, 555

Bacmann, A., Lefloch, B., Ceccarelli, C., Castets, A., Steinacker, J., \& Loinard, L 2002, A\&A, 389, 6

Bacmann, A., Lefloch, B., Ceccarelli, C., Steinacker, J., Castets, A., \& Loinard, L 2003, ApJ, 585, 55

Beichman, C. A., Myers P. C., Emerson, J. P., Harris, S., Mathieu, R., Benson, P. J., \& Jennings, R. E., 1986 ApJ, 307, 337

Belloche, A., \& André, P., Despois, D., \& Blinder, S. 2002 A\&A, 393, 927

Benson P.J., \& Myers P.C. 1989, ApJS, 71, 89

Black, J.H. 1994, in ASP Conf. Ser. 58, The First Symposium on the Infrared Cirrus and Diffuse Interstellar Clouds, ed. R.M. Cutri \& W. B. Latter (San Francisco: ASP), 355

Butner, H.M., Natta, A., \& Evans, N. J., II 1994, ApJ, 420, 326

Bourke, T. L., Crapsi, A., Myers, P. C., Evans, N. J., II, Wilner, D. J., Huard, T. L., Jørgensen, J. K., \& Young, C. H. 2005, ApJ, 633, L129

Bourke, T. L., Myers, P. C., Evans, N. J., II, Dunham, M. M., Kauffmann, J., Shirley, Y. L., Crapsi, A., Young, C. H., Huard, T. L., Brooke, T. Y., Chapman, N., Cieza, L., Lee, C. W., Teuben, P., \& Wahhaj, Z. 2006, ApJ 649, L37

Crapsi, A., Devries, C. H., Huard, T. L., Lee, J.-E., Myers, P. C., Ridge, N. A., Bourke, T. L., Evans, N. J., II, Jørgensen, J. K., Kauffmann, J., Lee, C. W., Shirley, Y. L., \& Young, C. H. 2005a, A\&A, 439, 1023

Crapsi, A., Caselli, P., Walmsley, C. M., Myers, P. C., Tafalla, M., Lee, C. W., \& Bourke, T. L. 2005b, ApJ, 619, 379 
Crapsi, A., van Dishoeck, E.F., Hogerheijde, M.R., Pontoppidan, K.M., \& Dullemond, C.P. 2008, A\&A, 486, 245

Dullemond, C.P., \& Dominik, C. 2004, A\&A, 417, 159

Dame, T. M., Ungerechts, H., Cohen, R.S., de Geus. E.J., Grenier, I.A., May, J., Murphy, D.C., Nyman, L.-A., \& Thaddeus, P. 1987, ApJ, 322, 706

Dame, T. M., Hartmann, D., Thaddeus, P. 2001, ApJ, 547, 792

de Geus, E. J., de Zeeuw, P. T., Lub, J. 1989, A\&A, 216, 44

Di Francesco, J., Evans, N. J., II, Caselli, P., Myers, P. C., Shirley, Y., Aikawa, Y., Tafalla, M. 2007, in Protostars and Planets V, ed. Reipurth, B., Jewitt, D. \& Keil K., (Tucson: University of Arizona Press), 17

Di Francesco, J., Johnstone, D., Kirk, H., MacKenzie, T., Ledwosinska, E., 2008, ApJS, 175, 277

Draine, B. T. 1978, ApJS, 36, 595

Drain, B.T., \& Lee, H. M. 1984, 285, 89

Dunham, M. M., Evans, N. J., II, Bourke, T. L., Dullemond, C. P., Young, C. H., Brooke, T. Y., Chapman, N., Myers, P. C., Porras, A., Spiesman, W., Teuben, P. J., Wahhaj, Z. 2006, ApJ, 651, 945

Dunham, M. M., Crapsi, A., Evans, N. J., II, Bourke, T. L., Huard, T.L., Myers, P.C., and Kauffmann, J. 2008, ApJS, in press, ArXiv e-prints, 806, arXiv:0806.1754

Enoch, M. L., Glenn, J., Evans, N. J., II, Sargent, A. I., Young, K. E., \& Huard, T. L. 2007, ApJ, 666, 982

Evans, N. J. II, Rawlings, J. M. C., Shirley, Y. L., Mundy, L. G. 2001, ApJ, 557, 193

Evans, N. J. II, Allen, Lori E., Blake, G. A., Boogert, A. C. A., Bourke, T., Harvey, P. M., Kessler, J. E., Koerner, D. W., Lee, C.W., Mundy, L. G., Myers, P. C., Padgett, D. L., Pontoppidan, K., Sargent, A. I., Stapelfeldt, K. R., van Dishoeck, E. F., Young, C. H., \& Young, K. E. 2003, PASP, 115, 965

Evans N.J. II., et al. 2007, Final Delivery of Data from the c2d Legacy Project: IRAC and MIPS (Pasadena: SSC), http://ssc.spitzer.caltech.edu/legacy/all.html

Fazio, G. G., et al. 2004, ApJS, 154, 10

Harvey, P., Merin, B., Huard, T. L., Rebull, L.M., Chapman, N., Evans II, N. J., \& Myers, P.C. 2007, ApJ, 663, 1149

Huard, T. L., et al. 2006, ApJ, 640, 391 
Ivezic, A., Nenkova, M., \& Elitzur, M. 1999, User Manual for DUSTY (Lexington: Univ. Kentucky)

Koo et al. 2002, JKAS, 36, 43

Lada, C. J. 1987, in IAU Symp. 115, Star forming regions, ed. Peimbert, M., \& Jugaku, J. (Dordrecht Reidel Publishing Co.), 1

Lada, C. J., \& Wilking, B. A. 1984, ApJ, 287, 610

Lada, C. J., Alves, J.F., \& Lombardi, M. 2007, in Protostars and Planets V, ed. Reipurth, B., Jewitt, D. \& Keil K., (Tucson: University of Arizona Press), 3

Lee, C. W., \& Myers, P.C. 1999, ApJS, 123, 233

Lee, C. W., Myers, P.C., \& Tafalla, M. 1999, ApJ, 526, 788

Löhr, A., Bourke, T.L., Lane, A.P., Myers, P.C., Parshley, S.C., Stark, A.A., \& Torhill, N.F.H. 2007, ApJS, 171, 478

Loinard, L., Torres, R. M., Mioduszewski, A., J., \& Rodriguez, L.F. 2008, ApJ, 675, L29

Lombardi, M., Lada, C.J., \& Alves, J. 2008, A\&A, 480, 785

Mamajek, E.E. 2008, Astronomische Nachrichten, 329, 10

Myers, P. C., Fuller, Gary A., Mathieu, R. D., Beichman, C. A., Benson, P. J., Schild, R. E., Emerson, J. P. 1987, ApJ, 319, 340

Myers, P. C., \& Ladd, E.F. 1993, ApJ, 413, 47

Ossenkopf, V., \& Henning, Th. 1994, A\&A, 291, 943

Padgett, D.L., et al. 2008, ApJ, 672, 1013

Rieke, G. H., et al. 2004, ApJS, 154, 25

Robitaille, T.P., Whitney, B.A., Indebetouw, R., Wood, K., \& Denzmore, P. 2006, ApJS, 167,256

Schechter, P. L., Mateo, M., \& Saha, A. 1993, PASP, 105, 1342

Shirley, Y. L., Nordhaus, M. K., Grcevich, J. M., Evans, N. J. II, Rawlings, J. M. C., \& Tatematsu, K. 2005, ApJ, 632, 982

Shu, F. H., Adams, F. C., Lizano, S. 1987, ARA\&A, 25, 23

Straižys, V., Černis, K.\& Bartašiūtè, S. 2003, A\&A, 405, 585

Stutz, A., Bourke, T.L., Rieke, G. H., Bieging, J. H., Misselt, K. A., Myers, P.C., \& Shirley, Y. L. 2008, arXiv:0811.1581v1

Tafalla, M., Myers, P.C., Caselli, P., Walmsley, C.M. \& Comito, C. 2002, ApJ, 569, 815 
Visser, A.E., Richer, J.S., \& Chandler, C.J. 2001, MNRAS, 323, 257

Visser, A.E., Richer, J.S., \& Chandler, C.J. 2002, AJ, 124, 2756

Ward-Thompson, D., Andre, P., Crutcher, R., Johnstone, D., Onishi, T., \& Wilson, C. 2007, in Protostars and Planets V, ed. Reipurth, B., Jewitt, D. \& Keil K., (Tucson: University of Arizona Press), 33

Wu, J., Dunham, M. M., Evans, N. J. II, Bourke, T. L., \& Young, C. H. 2007, AJ, 133, 1560

Young, C. H., et al. 2004, ApJS, 154, 396

Young. C. H., \& Evans, N. J. II. 2005, ApJ, 627, 293

Young, K. E., et al. 2006, ApJ, 644, 326 


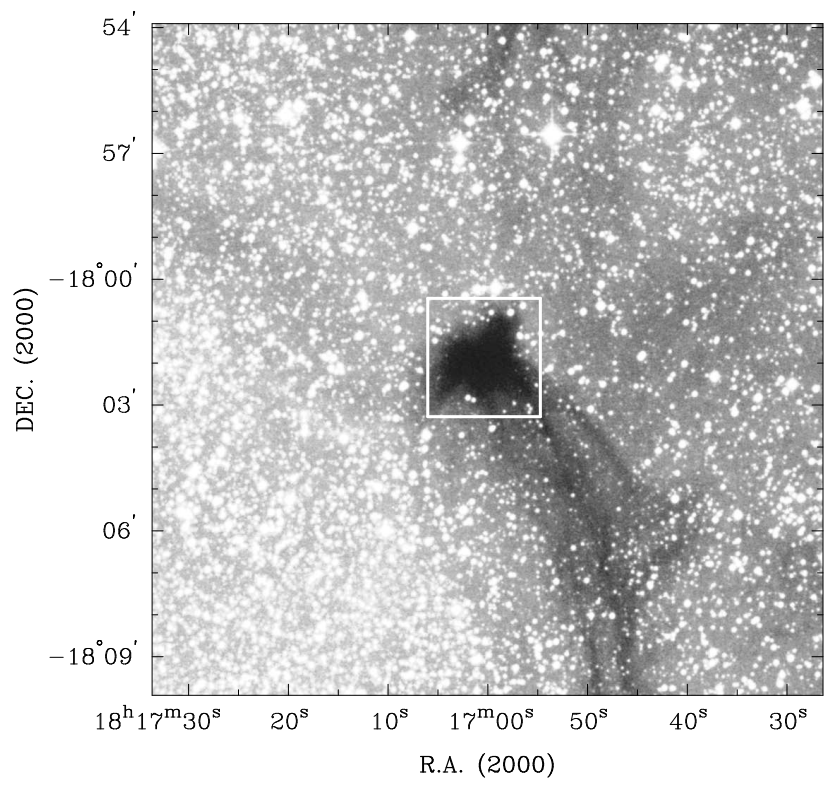

Fig. 1. - Optical DSS-red image around L328. L328 consists of a dark part of $\sim 2^{\prime} \times 2^{\prime}$ size and less opaque tails of $\sim 15$ arc-minute long extending to the SW. A white box indicates the inner part of the region observed by Spitzer for which the Spitzer images are shown in Fig. 2. 


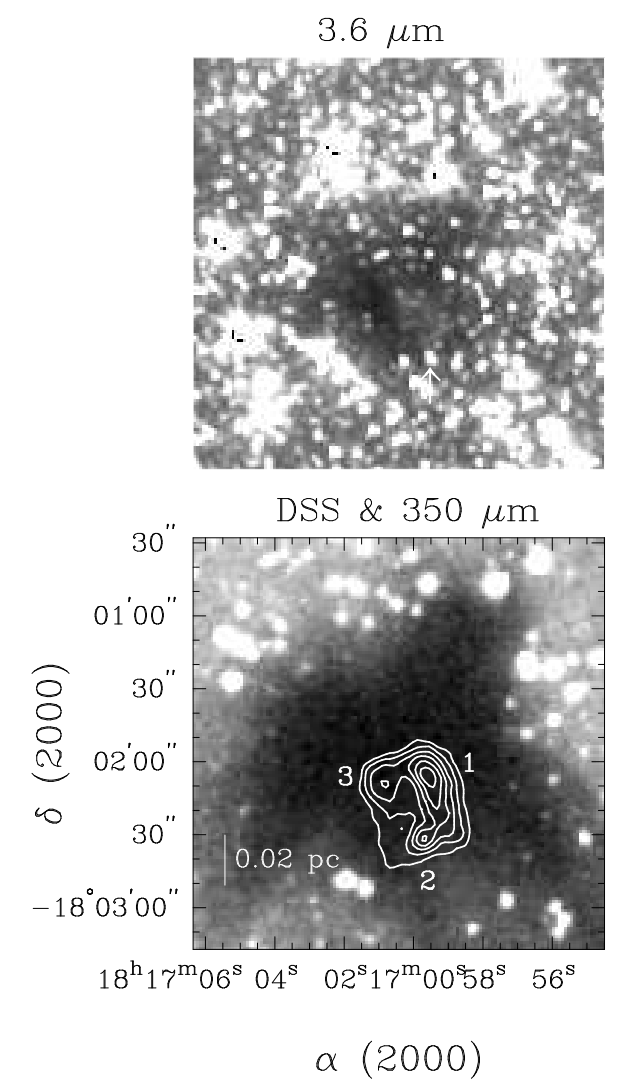

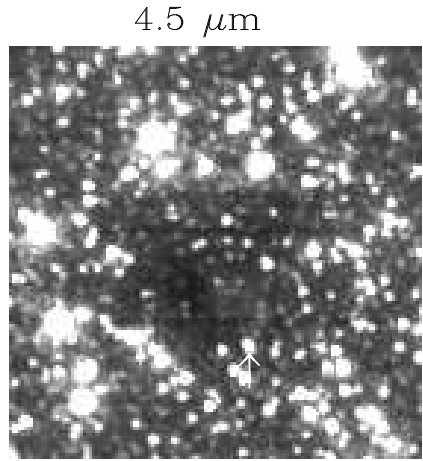

$24 \mu \mathrm{m}$
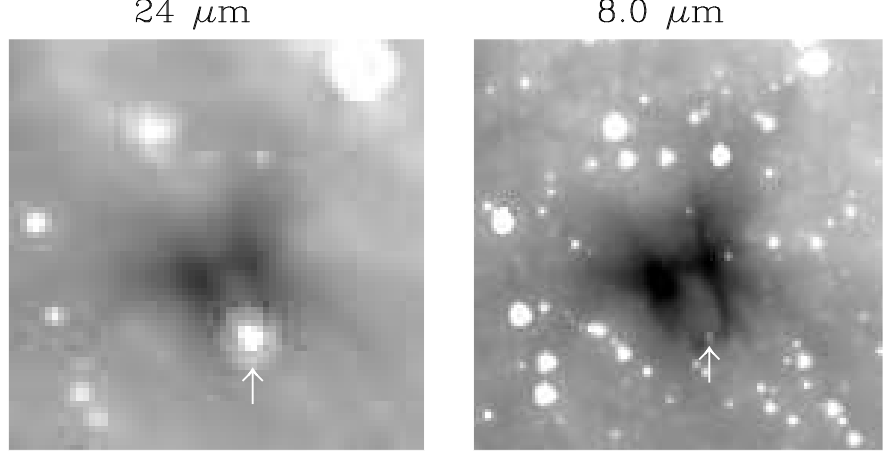

Fig. 2.- Multi-wavelength images of L328. In the bottom left panel is shown $350 \mu \mathrm{m}$ emission contours from Wu et al. (2007) over a DSS-red image of L328. Numbers indicate sub-cores, smm1, smm2, and smm3. The other panels show the Spitzer images of L328. The infrared source (L328-IRS) is indicated with an arrow. Intensities in all images are adjusted to best highlight the structures of the L328 subcores. The contour levels of $350 \mu \mathrm{m}$ emission are $7,13,19,25,31$, and $37 \mathrm{mJy} /$ beam. 

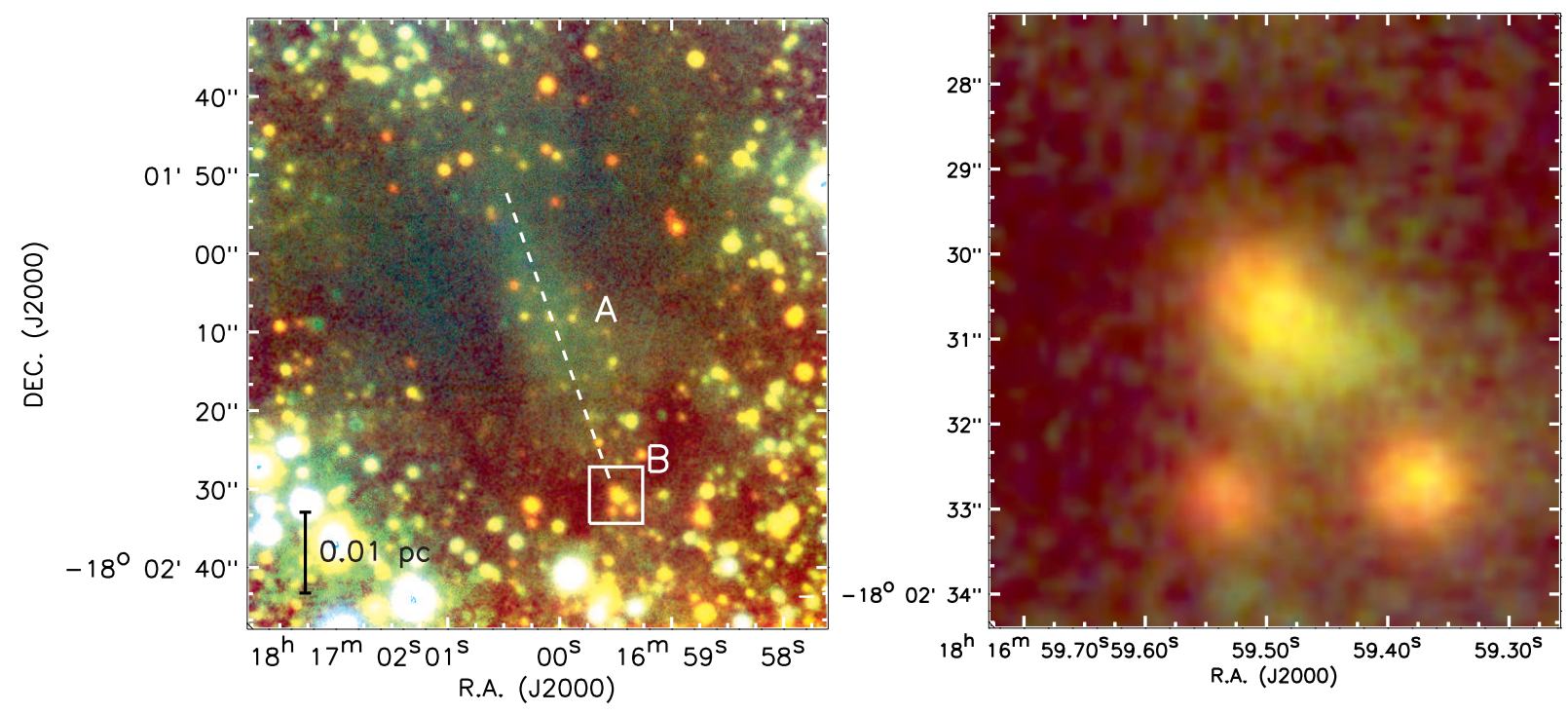

Fig. 3.- Near-IR composite images of L328. A left panel shows a 3 color JHKs composite image of L328 (blue $=\mathrm{J}$, green $=\mathrm{H}$, red $=\mathrm{Ks}$ ). This figure illustrates some structures possibly related to an outflow activity from L328-IRS, $\sim 30^{\prime \prime}$ cavity (designated as A) and nebulosity associated with L328-IRS itself (designated as B). A region drawn with a white box is enlarged in the right panel to show a close up of L328-IRS (at image center). Intensities in the composite images are adjusted to best enhance the structures of these cavity and nebulosity in L328 region. 


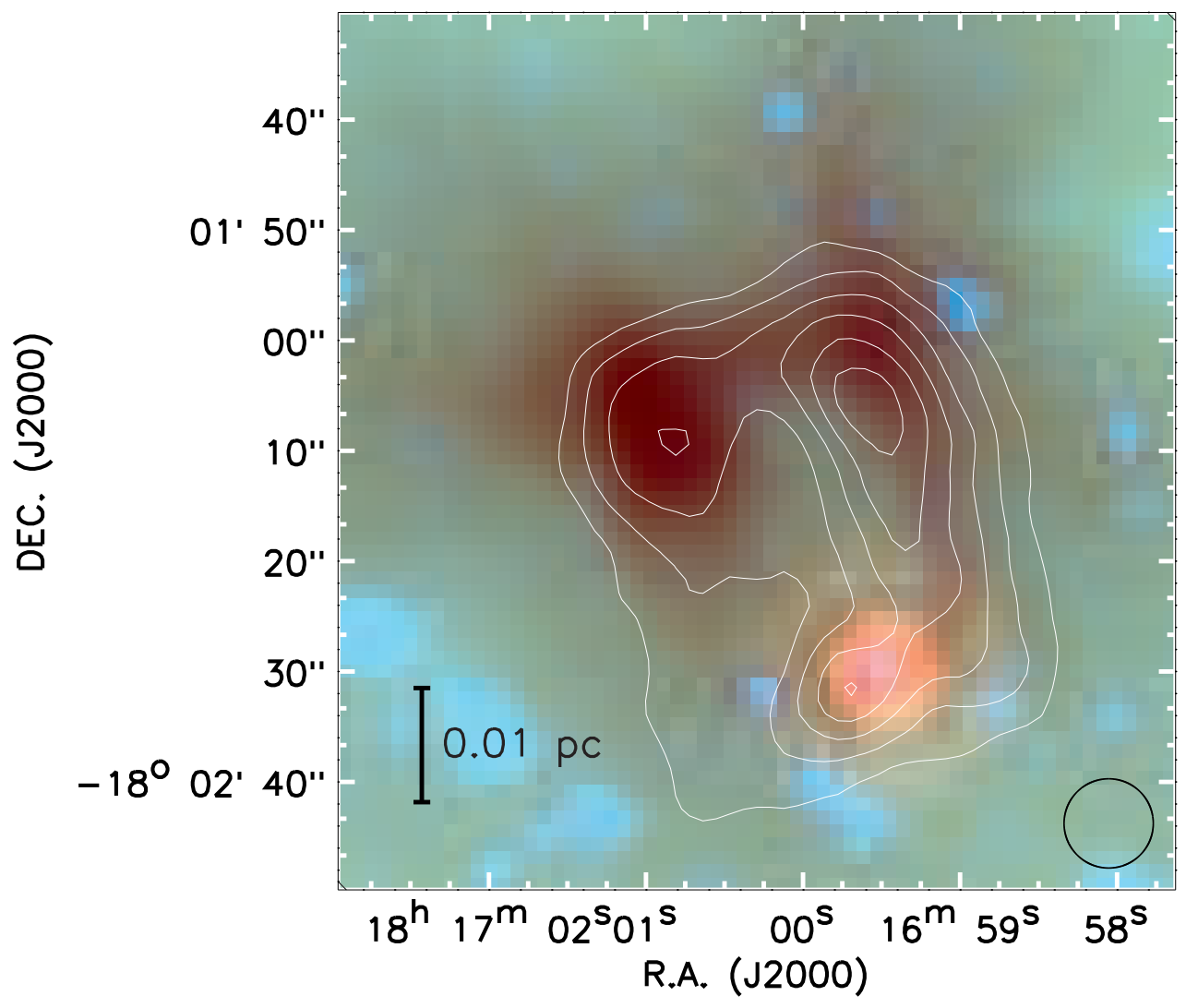

Fig. 4.- Three color composite image of L328 at $5.8 \mu \mathrm{m}$ (blue), $8 \mu \mathrm{m}$ (green), and $24 \mu \mathrm{m}$ (red). White contours are $350 \mu \mathrm{m}$ emission from Wu et al. (2007), drawn with the same levels as those in Fig. 2. A circle in the right corner indicates the FWHM beam size (8.5") of the $350 \mu \mathrm{m}$ data. Intensities are manually adjusted to show better the structures of the subcores and the $\sim 30^{\prime \prime}$ cavity in L328 region. 


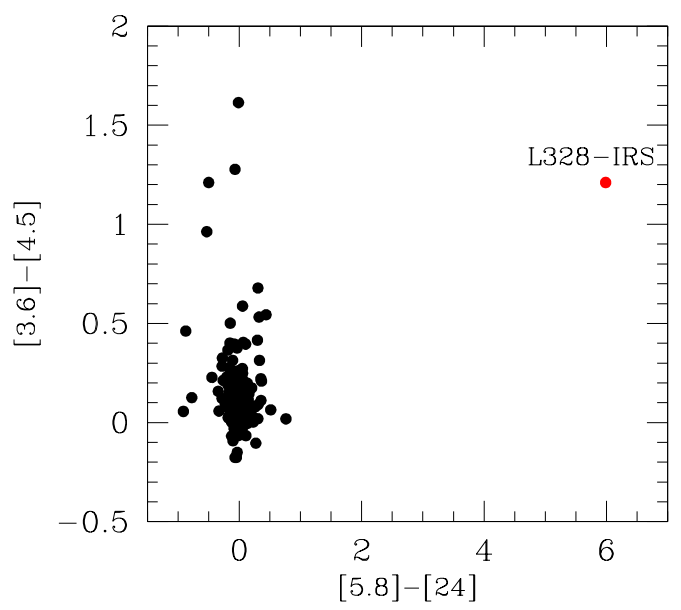

Fig. 5.- Spitzer color-color diagram of L328. Colors ([3.6]-[4.5] versus [5.8]-[24.0]) for all point sources in the L328 field observed with the Spitzer are displayed. 


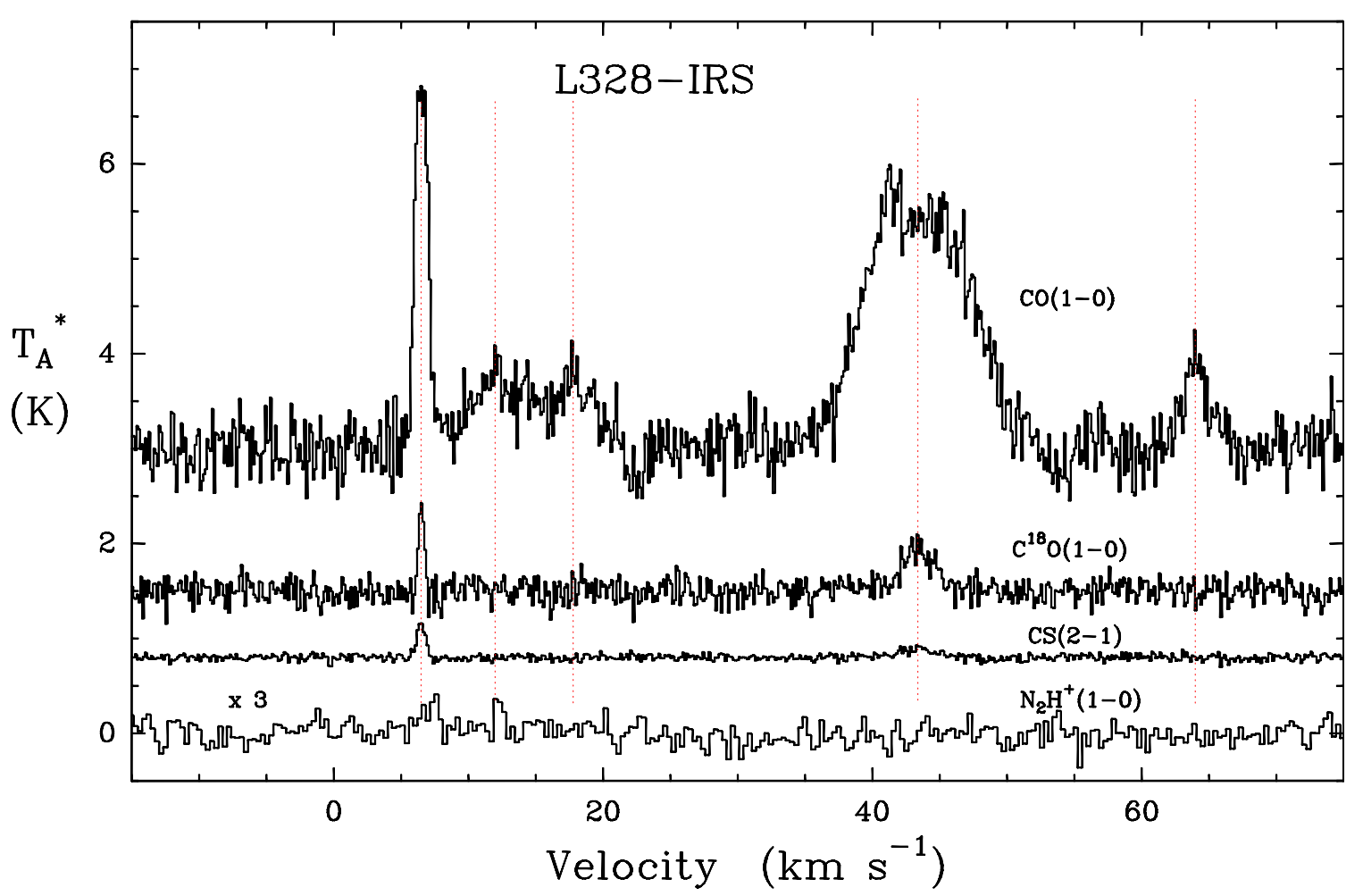

Fig. 6. - Molecular line spectra toward L328-IRS. Note that the position for the profiles $\left[(\alpha, \delta)_{J 2000}=\left(18^{\mathrm{h}} 16^{\mathrm{m}} 59.50,-18^{\circ} 02^{\prime} 03^{\prime \prime}\right)\right]$ is that of the CO emission peak and also the center of CO map in Fig. 7 , actually $\sim 27.5^{\prime \prime}$ offset from L328-IRS. Five different velocity components of $6.5,12.0,17.8,43.4$ and $64 \mathrm{~km} \mathrm{~s}^{-1}$ are indicated by vertically dotted lines. The temperature scale of $\mathrm{N}_{2} \mathrm{H}^{+}$(1-0) has been scaled up by a factor of 3 . 


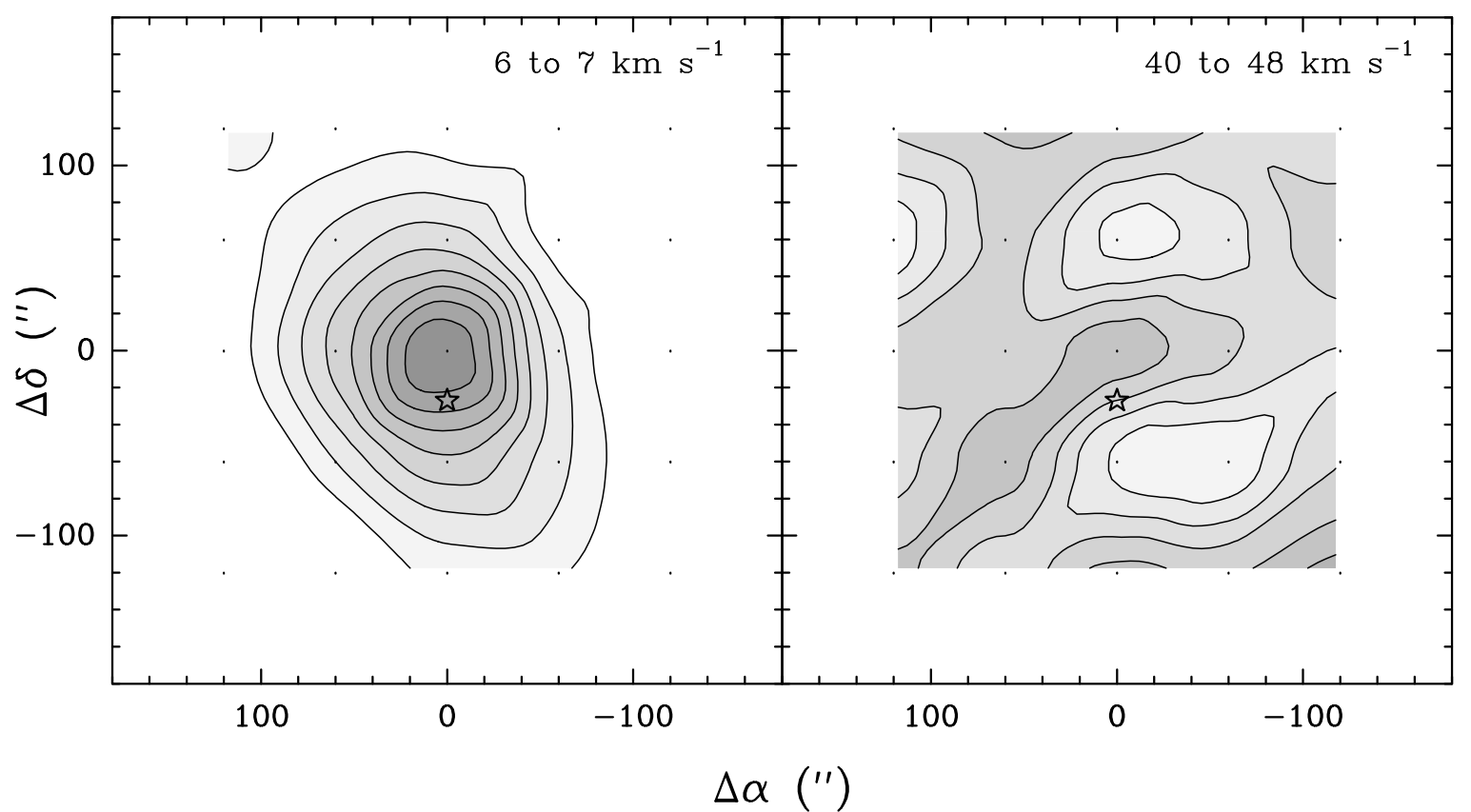

Fig. 7.- $\mathrm{C}^{18} \mathrm{O}$ integrated intensity $\left(\int \mathrm{T}_{\mathrm{A}}^{*} \mathrm{dv}\right)$ maps for two brightest velocity components. The central position of the map is $(\alpha, \delta)_{J 2000}=\left(18^{\mathrm{h}} 16^{\mathrm{m}} 59.50,-18^{\circ} 02^{\prime} 03^{\prime \prime}.\right)$, shifted by $\sim 27.5^{\prime \prime}$ from L328-IRS. The contour levels in the left panel are $0.10,0.15,0.20,0.25,0.30,0.35$, 0.40 , and $0.45 \mathrm{~K} \mathrm{~km} \mathrm{~s}^{-1}$ while the contour levels in the right panel are 1.0, 1.1, 1.2, and 1.3 $\mathrm{K} \mathrm{km} \mathrm{s}^{-1}$. A star in both panels marks the position of L328-IRS. 


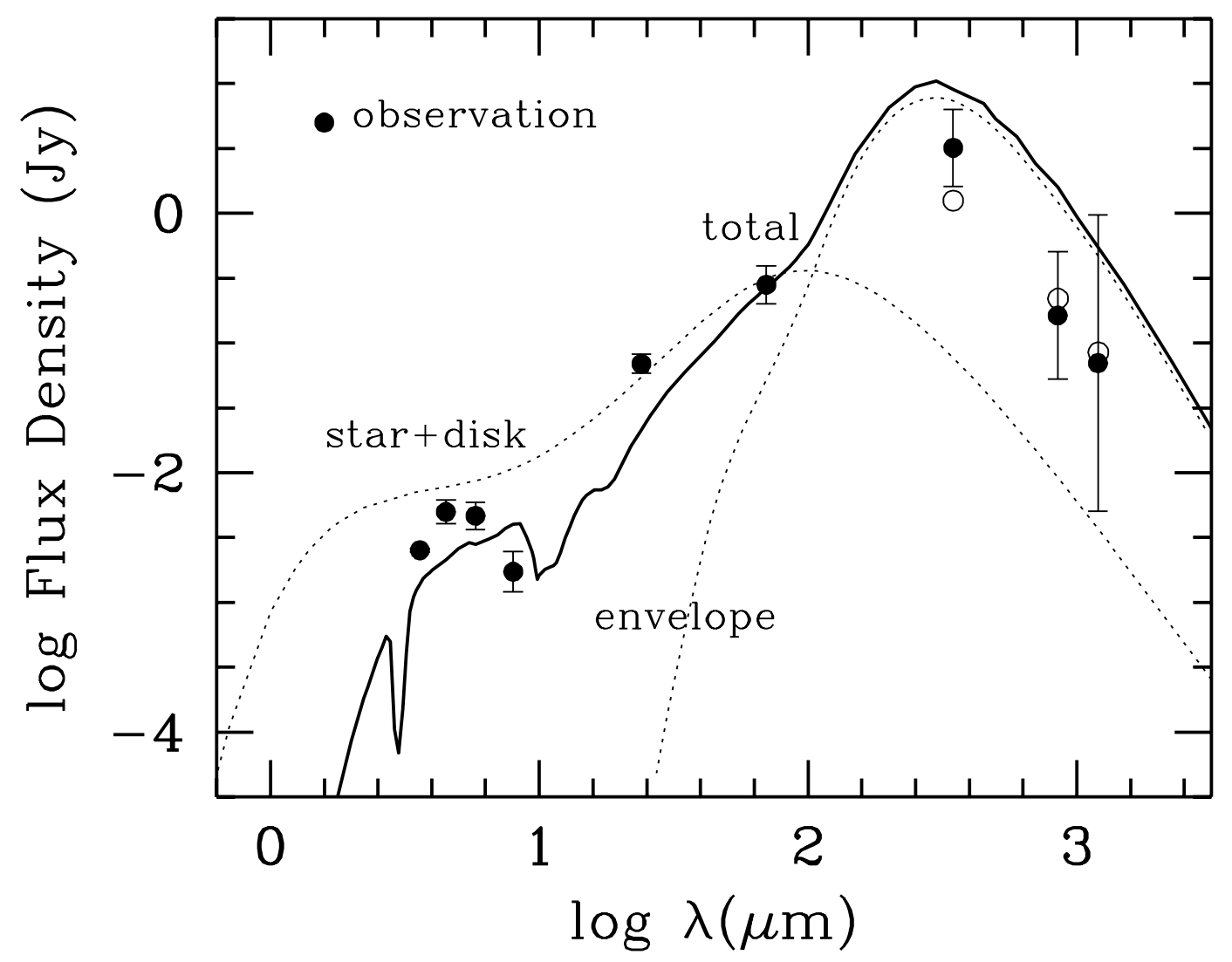

Fig. 8. - An example of a best fit radiative transfer model to the SED of L328-IRS. Filled circles with error bars are flux densities at the observed wavelengths. Open circles at 350 $\mu \mathrm{m}$ and long-ward are model fluxes measured with the same apertures as the observed ones. Our model consists of a stellar black body ( $\mathrm{T}=1500-3500 \mathrm{~K})$, a flared disk of $3.8 \times 10^{-4} \mathrm{M}_{\odot}$, and dusty isothermal envelope of inner radius $50 \mathrm{AU}$ and outer radius of 10,000 AU. The full model SED is shown with the solid continuous line, while the input internal star+disk and envelope models are shown as dotted lines and labeled. The input emission from the central source (star+disk) is absorbed by the dusty envelope at mainly short wavelengths (from NIR to $70 \mu \mathrm{m}$ ) so that the output emission at these wavelengths is dimmer than the input, and reprocessed in the envelope to produce emission at longer wavelengths. The model SED in the figure was obtained with $\mathrm{T}_{\text {star }}=2000 \mathrm{~K}$ and $\mathrm{L}_{\text {int }}=0.05 \mathrm{~L} \odot$. 
Table 1. Photometry of L328-IRS

\begin{tabular}{rrrr}
\hline \hline $\begin{array}{c}\text { Wavelength } \\
(\lambda)\end{array}$ & $\begin{array}{c}\text { Flux density } \\
(\mathrm{mJJ})\end{array}$ & $\begin{array}{c}\sigma \\
(\mathrm{mJy})\end{array}$ & $\begin{array}{c}\text { Aperture } \\
(\text { arcsec })\end{array}$ \\
\hline 3.6 & 2.52 & 0.34 & 9.0 \\
4.5 & 4.99 & 0.46 & 10.1 \\
5.8 & 4.64 & 0.48 & 10.1 \\
8.0 & 1.72 & 0.27 & 4.6 \\
24 & 69.2 & 5.0 & 34.3 \\
70 & 281 & 41 & 29.7 \\
$350^{\mathrm{a}}$ & 3200 & 950 & 20.0 \\
850 & 163 & 80 & 20.0 \\
1200 & 70 & 80 & 20.0 \\
\hline
\end{tabular}

aNote that this photometry was conducted by centering L328-IRS in an aperture of 20" and thus the flux density is larger than that (2000 mJy) reported by Wu et al. (2007) which had been obtained by centering on the peak position of the smm 2 core with the same aperture. 